Copyright (c) 2012 Universidad Tecnológica Equinoccial

ISSN: $1390-6542$

\title{
Microfiltración tangencial
}

Ing. Manuel Coronel ${ }^{1}$

\begin{abstract}
Resumen
El objetivo de este trabajo fue revisar los conceptos básicos y las aplicaciones de la tecnología de microfiltración tangencial (MFT) en la industria alimentaria, particularmente en jugos de fruta y derivados lácteos, entre otros. Se presenta, además, los tipos de membrana utilizadas en esta operación unitaria de separación. Finalmente, se resaltan brevemente los trabajos realizados en Ecuador con jugos de frutas locales y pretratamientos en leche de vaca.
\end{abstract}

\section{Palabras clave:}

Micro filtración tangencial, membrana, separación.

\begin{abstract}
The aim of this study was to review the basic concepts and applications of cross flow microfiltration technology (MFT) in the food industry, particularly in fruit juices and dairy products, among others. This review also presents the different types used in this unit operation. Finally, it briefly highlights the work done in Ecuador with native fruit juices and cow milk pretreatments.
\end{abstract}

Keywords:

Cross flow microfiltration, membrane, separation.

\section{Introducción}

Los procesos que usan membranas se originan inicialmente ante la necesidad de producir tecnologías más económicas y eficientes que las formas tradicionales de separación. Ejemplo de este desarrollo, lo representa la tecnología de osmosis inversa, a partir de la década del 60. Posteriormente, en los años 70 , se aplicó en otros sectores, entre ellos la industria alimentaria y biotecnológica, gracias a los nuevos materiales para membranas y equipamientos, así como la automatización de procesos (Saavedra \& Romero, 1999)

La microfiltración, al igual que todo proceso de separación que usa membranas, permite concentrar un líquido por retención de los componentes de mayor tamaño (retenido) respecto al diámetro del poro membranario. El líquido que atraviesa la membrana, denominado permeado, contiene los componentes o partículas de menor diámetro. Los diámetros de poro oscilan entre 0,1 y 10 micras, según el tipo de componente que se desee retener. El resto de métodos de filtración, como la ultrafiltración, nanofiltración u ósmosis inversa, usan membranas con diámetros de poro menor (Binetti, Bailo, \& Reinheimer, 2004).

\footnotetext{
${ }^{1}$ Universidad Tecnológica Equinoccial, Facultad de Ciencias de la Ingeniería, Quito - Ecuador (cfma7065@ute.edu.ec).
} 
La separación de partículas o de objetos macroscópicos se obtiene por filtración frontal. Las técnicas de filtración tangencial, que utilizan membranas artificiales, empiezan con la separación de partículas microscópicas o de especies biológicas tales como las bacterias, en cuyo caso se trata de microfiltración. Para especies o fragmentos de materias más pequeñas como los coloides, las macromoléculas o los virus, se trata de la ultrafiltración. Si se acompaña adecuadamente de un envasado aséptico, puede lograrse productos "esterilizados en frío". Esto permite hablar de una estabilidad microbiológica conseguida por mecanismos no térmicos (Cozzano, 2009). En cuanto a las pequeñas moléculas de masa molecular inferior a 1000 se separan por una técnica llamada nano filtración (Guizard, 1999).

\section{La Tecnología}

La micro filtración tangencial es un proceso de separación sólido-líquido de componentes de un fluido presurizado por medio de una membrana semipermeable (Vaillant, Pérez, \& Viquez, 2004).

Por tanto, la filtración tangencial es una técnica que se diferencia de la convencional, porque el flujo del material a filtrar, circula tangencialmente a la superficie membranaria. La tabla 1 indica los diferentes tipos de proceso de separación por medio de membranas, donde el principio impulsor o fuerza motriz de separación es la presión diferencial, el diámetro de poro que define la el tipo de componentes a separar que, además, se ejemplifican.

Tabla 1. Procesos de membrana (Pandolfi, 2008).

\begin{tabular}{|l|l|l|c|}
\hline \multicolumn{1}{|c|}{$\begin{array}{c}\text { Tipo de } \\
\text { proceso }\end{array}$} & Principio impulsor & $\begin{array}{c}\text { Diámetro de } \\
\text { poro }\end{array}$ & Ejemplos \\
\hline Microfiltración & Presión diferencial & $10-0,1 \mu \mathrm{m}$ & $\begin{array}{c}\text { Células microbianas, grandes coloides, } \\
\text { partículas pequeñas, etc. }\end{array}$ \\
\hline Ultrafiltración & Presión diferencial & $\begin{array}{l}<0.1 \mu \mathrm{m}-5 \\
\mathrm{~nm}\end{array}$ & $\begin{array}{c}\text { Proteínas, emulsiones, coloides } \\
\text { macromoleculares }\end{array}$ \\
\hline Nanofiltración & Presión diferencial & Aprox. 1 nm & Compuestos orgánicos y sales disueltas \\
\hline inversa & Presión diferencial & $<1 \mathrm{~nm}$ & $\begin{array}{r}\text { Pequeños compuestos orgánicos, sales } \\
\text { disueltas }\end{array}$ \\
\hline Electrodiálisis & $\begin{array}{l}\text { Potencial eléctrico } \\
\text { diferencial }\end{array}$ & $<5 \mathrm{~nm}$ & $\begin{array}{c}\text { Sales disueltas } \\
\text { Diálisis }\end{array}$ \\
\hline
\end{tabular}

A consecuencia de aplicar la presión (fuerza impulsora) los solutos próximos a la superficie se concentran en este sector, originando una resistencia extra al flujo y, por ende, disminuyendo el caudal (Ibañez, 2007). Los solutos retenidos por la membrana de microfiltración, que se 
encuentran en diámetros entre 0,1 y 10 micras, están representados por coloides, células microbianas y partículas pequeñas (Pandolfi, 2008). En la figura 1 se presenta una comparación esquemática entre la filtración convencional, denominada perpendicular (en relación a la orientación de la membrana) y la tangencial o paralela, porque la alimentación fluye paralela a la membrana.

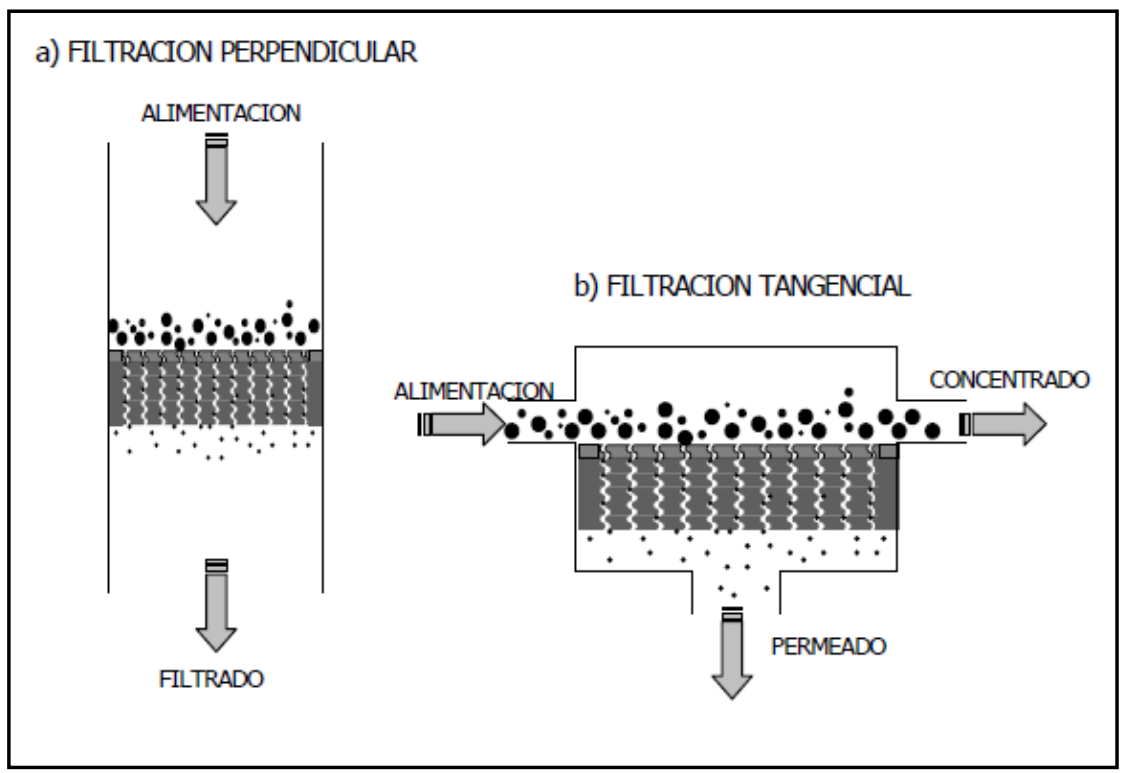

Figura 1. Filtración convencional o perpendicular (a) y tangencial o paralela (b) (Saavedra \& Romero, 1999).

En relación a las membranas porosas, estas pueden clasificarse, además, acorde con los materiales con los que han sido elaboradas: cerámicas, metálicas, vítreas, zeolíticas y de carbono. Últimamente, las membranas cerámicas, compiten por la gran cantidad de aplicaciones debido a su alta estabilidad, tanto térmica como química. Además, la facilidad de limpieza, permite un uso prolongado en el tiempo. Las membranas cerámicas son las que han provocado el uso en el mercado de las membranas inorgánicas en los últimos años. Esto se debe, especialmente, a sus aplicaciones en entornos químicamente diversos, altas temperaturas, presiones y costos de fabricación, que aunque son más altos que las poliméricas, resultan económicos para su uso durante periodos comparativamente prolongados de tiempo. El desarrollo de membranas cerámicas va orientado a satisfacer, los procesos de filtración de muchas industrias, principalmente la industria alimentaria, tratamiento de aguas y líquidos provenientes de fermentaciones y tratamientos de efluentes gaseosos, aunque es necesario mucho más esfuerzo para un total aprovechamiento de sus posibilidades (Benito, Conesa, \& Rodríquez, 2004).

La fuerza impulsora, para producir el efecto de separación, generalmente es la presión. La tabla 2 relaciona las presiones de trabajo para cada tipo de proceso de separación, la micro filtración presenta el menor nivel y los más altos flujos, mientras que, al otro extremo se encuentra la 
ósmosis inversa con valores que llegan a 100 bares de presión de trabajo, pero niveles de flujo menores, en relación a los demás métodos de separación.

Tabla 2. Presiones de trabajo en procesos de filtración mediante membranas. (Saavedra \& Romero, 1999)

\begin{tabular}{||c|c|c||}
\hline PROCESO & $\begin{array}{c}\text { PRESION } \\
\text { (Bar) }\end{array}$ & $\begin{array}{c}\text { FLUJO } \\
\left(\mathbf{L} / \mathbf{m}^{2} \mathbf{h}\right)\end{array}$ \\
\hline MICROFILTRACION & $0.1-5$ & $100-1000$ \\
ULTRAFILTRACION & $1-10$ & $10-200$ \\
NANOFILTRACION & $5-20$ & $10-100$ \\
OSMOSIS INVERSA & $10-100$ & $5-100$ \\
& & \\
\hline
\end{tabular}

El flujo de permeado tiende a decaer y es una de las principales dificultades en el proceso de micro filtración. La formación de una capa o torta en la superficie de la membrana también denominada colmatación, reduce la eficiencia del sistema. Esto se ha relacionado con el flujo reducido de permeado, resultado de cambios en las variables de operación (Ortiz, Vélez, \& Franco, 2008).

La colmatación, también denominada fouling, se genera por el depósito de precipitados, partículas, proteínas, sales, complejos macromoleculares, etc. Con los ciclos regulares de limpieza se logra que la membrana recupere sus propiedades originales (lbañez, 2007).

\section{Aplicaciones}

La filtración por membrana se utiliza para purificar o concentrar disoluciones y suspensiones o bien para fraccionar una mezcla de varios solutos y se puede utilizar como alternativa a la floculación, las técnicas de purificación de sedimentos, la adsorción (filtros de arena y filtros de carbón activado, intercambiadores iónicos), extracción o destilación (Sanchez, 2007).

Existe un sinnúmero de ejemplos que demuestran la aplicación de la tecnología membranaria en la industria de alimentos.

- Procesamiento de leche entera micro filtrada. Esto permite eliminar más del $99 \%$ de bacterias sin comprometer las propiedades físicas, químicas y sensoriales hasta 15 días a 6ํㅡ. Más del 99\% de las proteínas lácteas logran atravesar la membrana (Binetti, et al., 2004).

- Agua para bebidas. Aquí básicamente se purifica el agua para usos múltiples (Prieto, Carrillo, \& Rodríguez, 2005). 
- Clarificación de jugos naturales. Por ejemplo, jugos de mora, tomate de árbol, naranjilla, entre otros (Vasco, 2008).

- Clarificación de vinos. Se ha logrado eliminar considerablemente microorganismos contaminantes y levaduras propias de la fermentación original. Esta operación es usual en países con tradición en la producción de vinos, especialmente tintos (Pandolfi, 2008).

En Ecuador, las investigaciones al respecto se han orientado en mayor medida en jugos de fruta y suero lácteo, como se aprecia en la tabla 3. Los trabajos, en relación al componente retenido del proceso de micro filtración, apuntan además al aprovechamiento de componentes bioactivos.

Tabla 3. Trabajos con microfiltración tangencial en Ecuador (<10 años).

\begin{tabular}{|c|c|c|}
\hline Artículo & Aplicación & Resultado \\
\hline \multicolumn{3}{|l|}{ Frutas } \\
\hline $\begin{array}{l}\text { Brito, Picho, Vera \& Vaillant } \\
(2010)\end{array}$ & Granadilla & Clarificación \\
\hline León \& Rosero (2009) & Uvilla & Clarificación \\
\hline Laverde (2010) & Arazá & Clarificación \\
\hline Yánez (2006) & Piña & Bebida gaseosa \\
\hline Vasco (2008) & $\begin{array}{l}\text { Mora, tomate de árbol y } \\
\text { naranjilla }\end{array}$ & $\begin{array}{c}\text { Proyecto de producción } \\
\text { industrial }\end{array}$ \\
\hline $\begin{array}{l}\text { Tupuna, Vera \& Ruales(2011) } \\
\text { Lácteos }\end{array}$ & Mortiño & Clarificación \\
\hline Camacho (2009) & Suero lácteo & $\begin{array}{l}\text { Recuperación de } \\
\text { concentrado proteico }\end{array}$ \\
\hline Arias \& Espinel (2006) & Leche para quesos & $\begin{array}{l}\text { Lacto suero concentrado y } \\
\text { rendimiento quesero }\end{array}$ \\
\hline Miranda \& Cuaspud (2011) & Leche concentrada & Manjar blanco \\
\hline
\end{tabular}

\section{Conclusión}

La tecnología de microfiltración tangencial permite obtener productos de alta calidad, especialmente organoléptica, microbiológica y funcional. Se ha transformado en una línea para obtención de compuestos bioactivos y representa una alternativa interesante para procesar frutas exóticas. 


\section{Bibliografía}

Arias, M., \& Espinel, A. (2006). Evaluación de la microfiltración tangencial para fabricación de queso y aprovechamiento de lactosuero. EPN, Quito.

Benito, J., Conesa, A., \& Rodríquez, M. (2004). Membranas cerámicas. Tipos, métodos de obtención y caracterización. Boletín de la sociedad española del vidrio y cerámica (CSIC), $43(5)$,

Binetti, A., Bailo, N., \& Reinheimer, J. (2004). Aplicaciones de la Microfiltración en la industria láctea. Mundo Lácteo, 10-14. Retrieved from http://www.alimentariaonline.com/apadmin/img/upload/MLC002_microfiltracionWSF.pdf

Brito, B., Picho, L., Vera, E., \& Vaillant, F. (2010). Estudio de las Condiciones Óptimas de Operación para la Obtención de Jugo Clarificado de Granadilla (Passiflora Ligularis L.) a través de la Microfiltración Tangencial. revista Tecnológica ESPOL - RTE, 23(2), 49-55.

Camacho, M. (2009). Obtención de un concentrado proteico de suero de leche de vaca utilizando tecnología de membranas. EPN, Quito.

Cozzano, S. (2009). IMPACTO DEL PROCESO DE MICROFILTRACIÓN TANGENCIAL SOBRE LOS COMPUESTOS ANTIOXIDANTES EN JUGOS DE FRUTAS. Santiago: Proyecto PAVUC - Universidad de Chile.

Guizard, C. (1999). Técnicas membranarias de filtración de líquidos. Micro-, Ultra-, Nanofiltración y Ósmosis Inversa. Mérida.

Ibañez, R. (2007). ESTUDIO DE LA ULTRAFILTRACIÓN DE PROTEÍNAS MODELO CON MEMBRANAS CERÁMICAS. Universidad de Granada, Granada.

Laverde, J. (2010). Estudio de las condiciones óptimas para la obtención de jugo clarificado de Arazá (Eugenia stipitata), mediante proceso enzimático y membranario. EPN, Quito.

León, G., \& Rosero, E. (2009). OBTENCIÓN DE JUGO CLARIFICADO DE UVILLA (Physalis peruviana. L.), UTILIZANDO DEGRADACIÓN ENZIMÁTICA Y MICROFILTRACIÓN TANGENCIAL. UTN, Ibar5ra.

Miranda, M., \& Cuaspud, J. (2011). Elaboración de manjar blanco saborizado, utilizando leche concentrada por microfiltración tangencial reconstituida con grasa vegetal y animal. UTN, lbarra.

Ortiz, M., Vélez, C., \& Franco, E. (2008). modelos matemáticos de colmatación de membranas en microfiltración tangencial. Revista Ingeniería e Investigación, 28(1).

Pandolfi, E. (2008). Aplicación de la tecnología de membranas en el proceso de vinificación., Universidad de Concepción, Santiago.

Prieto, M., Carrillo, A., \& Rodríguez, M. (2005). Ensayos preliminares de microfiltración directa para potabilización de aguas superficiales en la Sabana de Bogotá. Revista de Ingeniería(22).

Saavedra, A., \& Romero, J. (1999). ASPECTOS GENERALES SOBRE PROCESOS Y TECNOLOGIAS DE MEMBRANAS (DOCUMENTO 1). Grupo de procesos de membranas,

Sanchez, F. (2007). Estudio y diseño de una planta de producción de membranas cerámicas de coste reducido. Universidad Barcelona. 
Tupuna, S., Vera, E., \& Ruales, J. (2011). OBTENCIÓN DE JUGOS CLARIFICADOS DE MORTIÑO (Vaccinium floribundum Kunth) MEDIANTE EL USO DE TECNOLOGÍA DE MEMBRANAS. DECAB - EPN.

Vaillant, F., Pérez, A., \& Viquez, F. (2004). Microfiltración tangencial: una alternativa innovadora para la transformación de frutas tropicales. La Alimentación Latinoamericana(252), 38-46.

Vasco, A. (2008). Estudio de prefactibilidad para la instalación de una planta productora de jugos clarificados y jugos clarificados concentrados de mora, tomate de árbol y naranjilla, utilizando la tecnología de membranas. EPN, Quito.

Yánez, R. (2006). Desarrollo de una bebida gaseosa utilizando jugo clarificado de piña (variedad cayena champaca) por microfiltración tangenc. EPN, Quito. 